\title{
Value of Real-Time Bedside Ultrasonography in the Etiologic Diagnosis of Acute Dyspnea
}

\author{
Ning Xu, Zhangshun Shen, Chang Lv, Qian Zhao, Hui Guo, Huiling Zhang, Zhichao Ma, Jianguo Li* \\ Department of Emergency, Hebei General Hospital, Shijiazhuang, China \\ Email: *lijg65@163.com
}

How to cite this paper: $\mathrm{Xu}, \mathrm{N}$., Shen, Z.S., Lv, C., Zhao, Q., Guo, H., Zhang, H.L., Ma, Z.C. and Li, J.G. (2021) Value of Real-Time Bedside Ultrasonography in the Etiologic Diagnosis of Acute Dyspnea. International Journal of Clinical Medicine, 12, 441-450. https://doi.org/10.4236/ijcm.2021.1210040

Received: September 24, 2021

Accepted: October 18, 2021

Published: October 21, 2021

Copyright (c) 2021 by author(s) and Scientific Research Publishing Inc. This work is licensed under the Creative Commons Attribution International License (CC BY 4.0).

http://creativecommons.org/licenses/by/4.0/

\begin{abstract}
Objective: To explore the value of real-time bedside ultrasonography in the etiologic diagnosis of acute dyspnea. Methods: Sixty-two patients with acute dyspnea who were treated in our hospital from January 2016 to December 2020 were randomly selected and their clinical data were retrospectively analyzed. All patients were randomly divided into a control group for routine examinations $(\mathrm{n}=31)$ and an observation group for real-time beside ultrasonography $(\mathrm{n}=31)$. The costs of medical examinations, examination duration, and diagnostic results of severe pneumonia, acute cardiogenic pulmonary edema, pulmonary embolism, chronic obstructive pulmonary disease, and pneumothorax (including sensitivity, specificity, positive predictive value, negative predictive value, and diagnostic accuracy) of the two groups of patients were compared and analyzed. Results: Compared with the control group, the observation group had significantly shorter examinations $(\mathrm{P}<0.05)$. Although the cost of medical examinations of the observation group tended to be higher, the difference between groups was not significant $(P>0.05)$. Moreover, there were no significant differences in left ventricular ejection fraction, left ventricular end-diastolic diameter, or brain natriuretic peptide between the two groups $(\mathrm{P}>0.05)$. Comparison of the etiologic diagnosis results between the two groups showed that the observation group had significantly higher diagnostic sensitivity, specificity, positive and negative predictive values, and diagnostic accuracy for various causes compared with the control group $(\mathrm{P}<$ 0.05). Conclusion: Real-time bedside ultrasonography for the etiologic diagnosis of patients with acute dyspnea was quicker and had higher diagnostic accuracy; thus providing accurate guidance for the disease treatment, and having a higher promotional value in clinical practice compared with routine examinations.
\end{abstract}

\section{Keywords}

Real-Time Bedside Ultrasonography, Acute Dyspnea, Etiological Diagnosis, 


\section{Introduction}

Dyspnea is relatively common in clinical practice, especially in critically ill patients in emergency departments. Dyspnea triggers many factors, including toxicity, hematogenous, cardiogenic, pulmonary, and neuropsychiatric factors. Dyspnea in patients who fail to receive timely and effective treatment are gradually aggravated with prolonged treatment, often leading to death in a short time. Hence, timely and accurate evaluation of the disease is key to the successful treatment of patients with dyspnea and the formation of corresponding treatment regimens based on the evaluation results [1]. Computed tomography (CT), biochemical blood tests, and X-ray examinations are common methods for the diagnosis and detection of the causes of acute dyspnea. Despite their relatively good diagnostic efficacy, these methods cannot be conducted at the bedside, especially during the process of hospital transferring and examinations of the patients [2]. With the continuous development of clinical diagnostic technology and the continuous updating and application of diagnostic equipment in China, bedside ultrasonography has been extensively used in the etiological diagnosis of acute dyspnea patients in clinical practice, and its effects have been highly recognized by both medical professionals and patients [3]. Real-time bedside ultrasonography facilitates faster, simpler, and more accurate diagnosis, helping clinicians to quickly analyze the causes of patient's illness. Real-time bedside ultrasonography is a noninvasive examination that can monitor a patient's conditions in real time without causing radiation damage to the patient [4]. In the current study, we selected patients with acute dyspnea who were treated in our hospital in the past 3 years, retrospectively analyzed their clinical data, and listed the diagnostic value of real-time beside ultrasonography on the causes of acute dyspnea.

\section{Information and Methods}

\subsection{General Information of the Patients}

Sixty-two patients with acute dyspnea who were treated in our hospital from January 2016 to December 2020 were randomly selected and their clinical data were retrospectively analyzed. The inclusion criteria were as follows: 1) patients who met the diagnostic criteria of acute dyspnea [5] and were confirmed to have acute dyspnea; 2) patients with complete clinical data; and 3) patients whose family members were informed of this study. The exclusion criteria of this study were as follows: 1) patients with contraindications to real-time bedside ultrasonography for the diagnosis; 2) patients with severe infectious diseases; and 3) with mental illness. All patients were randomly divided into a control group ( $\mathrm{n}=$ $31)$ and an observation group $(\mathrm{n}=31)$. The observation group comprised 21 
males and 10 females, with the youngest age of 27 years old and the oldest age of 68 years old (mean age: $55.38 \pm 5.81$ years old). Among the patients of the observation group, 4 patients had acute cardiogenic pulmonary edema, 8 patients had severe pneumonia, 8 patients had chronic obstructive pulmonary disease, 5 patients had pulmonary embolism, and 6 patients had pneumothorax. The control group comprised 22 males and 9 females, with the youngest age of 28 years old and the oldest age of 28 years old (mean age: $55.08 \pm 5.01$ years old). Among the control group, 5 patients had acute cardiogenic pulmonary edema, 9 patients had severe pneumonia, 8 patients had chronic obstructive pulmonary disease, 4 patients had pulmonary embolism, and 5 patients had pneumothorax. The research protocol of this study was approved by the ethics committee of our hospital. No significant difference in general information was found between the two groups $(\mathrm{P}>0.05)$.

\subsection{Methods}

The diagnostic criteria of dyspnea included abnormal changes in the heart shadow in X-ray, rales at the bottom of the lungs, and a significant change in pulmonary rales when changing the body position of the patients. The severity of dyspnea was based on the degrees of damage during gas exchange and the breathing pattern of the patients.

The control group received routine examinations, including chest X-ray, measurement of brain natriuretic peptide (BNP), analysis of arterial blood gas, various physical examinations, troponin (TNI) test, and routine blood tests. A comprehensive analysis based on the results of each examination was performed by physicians of each department before preparing the corresponding treatment regimens for individual patients. Elbow venous blood $(5 \mathrm{ml})$ was collected from each patient and placed in a collecting tube containing ethylenediaminetetraacetic acid (EDTA) anticoagulant. The blood sample was centrifuged at $3000 \mathrm{rpm}$ for 15 min before collecting the serum to detect BNP by enzyme-linked immunosorbent assay. The left ventricular ejection fraction (LVEF) and left ventricular end-diastolic diameter (LVED) of the patients were detected by conventional electrocardiogram before recording the details.

The observation group received real-time bedside ultrasonography. In brief, a color Doppler ultrasound diagnostic apparatus (Kaili portable color Doppler Elexp) and its matching three-dimensional ultrasound probes, $4 \mathrm{~V}$ and MSS probes, were used for lung ultrasonography by adjusting the frequency of the $4 \mathrm{~V}$ and MSS probes to $1.5 \mathrm{MHz}-4.0 \mathrm{MHz}$ and $2.0 \mathrm{MHz}-4.0 \mathrm{MHz}$ in each patient in the supine position. Lung ultrasonography was conducted by a physicians who did not participate in the diagnosis and treatment of the patient. The results were analyzed by the attending physician to devise the appropriate treatment regimens for each patient. The BNP level was detected by a rapid beside test for BNP, and the LVEF and LEVD were measured by real-time bedside ultrasonography before recording the details. 


\subsection{Observation Indicators}

The costs of the medical examinations; the examination duration; the diagnostic results of severe pneumonia, acute cardiogenic pulmonary edema, pulmonary embolism, chronic obstructive pulmonary disease, and pneumothorax (including sensitivity, specificity, positive predictive value, negative predictive value, and diagnostic accuracy); and the LVEF, LVED, and BNP of the patients in the two groups were recorded and statistically analyzed. The sensitivity, specificity, positive predictive value, negative predictive value, and diagnostic accuracy were determined using the following equations:

Sensitivity $=$ number of true positive cases/(number of true positive cases + number of false negative cases) $\times 100 \%$

Specificity $=$ number of true negative cases/(number of true negative cases + number of false positive cases) $\times 100 \%$

Negative predictive value $=$ number of true negative cases $/$ (number of true negative cases + number of false negative cases) $\times 100 \%$

Positive predictive value $=$ number of true positive cases $/$ (number of true positive cases + number of false positive cases) $\times 100 \%$

Accuracy rate $=$ (number of true positive cases + number of true negative cases)/total number of patients $\times 100 \%$.

\subsection{Statistical Analysis}

The SPSS 20.0 software package (IBM, Armonk, NY) was used to perform all statistical analyses. The results of the examination duration, cost of medical examinations, and levels of LVEF, LVED, and BNP are presented as $(\bar{x} \pm s)$, and a $\mathrm{t}$-test was used to compare the differences between the groups. The sensitivity, specificity, positive predictive value, negative predictive value, and the rate of diagnostic accuracy are presented as (n, \%), and the chi-square test was used to compare the differences between the two groups. A P-value $<0.05$ was considered to indicate a significant difference.

\section{Results}

\subsection{Examination Duration and Costs of Medical Examinations}

Comparison of the examination duration between the two groups showed that the duration of examination in the control group (46.89 $\pm 5.82 \mathrm{~min})$ was significantly longer than that of the observation group $(19.21 \pm 2.65 \mathrm{~min})(\mathrm{P}<0.05)$. Although the cost of medical examination in the observation group (CN¥785.05 \pm 8.05 ) tended to be higher than that of the control group (CN¥781.00 \pm 7.03 ), no significant difference was found between the two groups $(\mathrm{P}>0.05$, see Table 1 for details).

\subsection{LVEF, LVED, and BNP Levels}

As shown in Table 2, no significant differences in the levels of LVEF, LVED, or BNP were found between the two groups of patients. 
Table 1. Comparison of the examination duration and cost of medical examinations between the two groups.

\begin{tabular}{ccc}
\hline Group & Examination duration $(\mathrm{min})$ & Cost of medical examination (CN¥) \\
\hline Observation group $(\mathrm{n}=31)$ & $19.21 \pm 2.65$ & $785.05 \pm 8.05$ \\
Control group $(\mathrm{n}=31)$ & $46.89 \pm 5.82$ & $781.00 \pm 7.03$ \\
$\mathrm{~T}$ & 13.841 & 2.346 \\
$\mathrm{P}$ & $<0.001$ & 0.047 \\
\hline
\end{tabular}

Table 2. Comparison of LVEF, LVE, and BNP levels between the two groups.

\begin{tabular}{cccc}
\hline Group & LVEF $(\%)$ & LVED $(\mathrm{m})$ & BNP $(\mathrm{ng} / \mathrm{L})$ \\
\hline Observation group $(\mathrm{n}=31)$ & $48.21 \pm 5.65$ & $57.05 \pm 5.25$ & $275.33 \pm 54.25$ \\
Control group $(\mathrm{n}=31)$ & $47.89 \pm 5.82$ & $56.34 \pm 5.64$ & $278.34 \pm 55.64$ \\
$\mathrm{t}$ & 1.041 & 1.146 & 1.384 \\
$\mathrm{P}$ & 0.102 & 0.113 & 0.135 \\
\hline
\end{tabular}

\subsection{Results of Etiologic Diagnosis in the Two Groups}

As shown in Tables 3-7, comparison of the diagnostic results of the two groups showed that the observation group had higher diagnostic sensitivity, specificity, positive predictive value, negative predictive value, and diagnostic accuracy for various causes of acute dyspnea than the control group $(\mathrm{P}<0.05)$.

\section{Discussion}

Dyspnea is relatively common in clinical practice, especially in the emergency department, and represents one of the main complaints of critically ill patients. With the gradual increase in the clinical research, data have shown that more than 20 million patients in the emergency department complain of respiratory tract dysfunction annually. Moreover, patients who present to the emergency department with dyspnea as the main complaint account for approximately $5 \%$ of the total number of patients in the emergency department [6]. Continuous improvement and optimization of existing diagnoses and treatment regimens greatly reduce the mortality of patients with acute dyspnea. X-ray and CT are commonly used for the etiologic diagnosis of acute dyspnea. Although X-ray diagnosis can be performed at the bedside, the value of imaging data obtained is relatively low and does not support the whole process of diagnosis and treatment, resulting in limited application [7]. Although the imaging data obtained by CT for the diagnosis are relatively abundant, CT cannot be performed at the bedside and requires patients to be transferred from the ward to the CT room for the examination and then transferred back from the CT room to the ward. The transportation of patients for CT and back is extremely risky for critically ill patients [8].

Recently, with the continuous development of ultrasound diagnostic technology and the continuous updating and upgrading of ultrasound diagnostic equipment 
Table 3. Comparison of the diagnostic results of severe pneumonia based on the two diagnostic methods (\%).

\begin{tabular}{cccccc}
\hline Group & Sensitivity & Specificity & Positive predictive value & Negative predictive value & Diagnostic accuracy \\
\hline Observation group $(\mathrm{n}=8)$ & $87.50(7 / 8)$ & $40.00(2 / 5)$ & $77.78(7 / 9)$ & $80.00(4 / 5)$ & $69.23(9 / 13)$ \\
Control group $(\mathrm{n}=9)$ & $62.50(5 / 8)$ & $20.00(1 / 5)$ & $55.56(5 / 9)$ & $40.00(2 / 5)$ & $46.15(6 / 13)$ \\
$\chi^{2}$ & 9.045 & 9.684 & 10.684 & 19.557 & 11.531 \\
$\mathrm{P}$ & $<0.001$ & $<0.001$ & $<0.001$ & $<0.001$ & $<0.001$ \\
\hline
\end{tabular}

Table 4. Comparison of the diagnostic results of acute cardiogenic pulmonary edema based on the two diagnostic methods.

\begin{tabular}{cccccc}
\hline Group & Sensitivity & Specificity & Positive predictive value & Negative predictive value & Diagnostic accuracy \\
\hline Observation group $(\mathrm{n}=4)$ & $60.00(3 / 5)$ & $66.67(2 / 3)$ & $60.00(3 / 5)$ & $75.00(3 / 4)$ & $62.50(5 / 8)$ \\
Control group $(\mathrm{n}=5)$ & $40.00(2 / 5)$ & $33.33(1 / 3)$ & $40.00(2 / 5)$ & $50.00(2 / 4)$ & $37.50(3 / 8)$ \\
$\chi^{2}$ & 8.066 & 12.012 & 9.043 & 12.005 & 12.981 \\
$\mathrm{P}$ & 0.005 & $<0.001$ & $<0.001$ & $<0.001$ & $<0.001$ \\
\hline
\end{tabular}

Table 5. Comparison of the diagnostic results of pulmonary embolism based on the two diagnostic methods.

\begin{tabular}{cccccc}
\hline Group & Sensitivity & Specificity & Positive predictive value & Negative predictive value & Diagnostic accuracy \\
\hline Observation group $(\mathrm{n}=5)$ & $66.67(4 / 6)$ & $66.67(2 / 3)$ & $71.43(5 / 7)$ & $60.00(3 / 5)$ & $66.67(6 / 9)$ \\
Control group $(\mathrm{n}=4)$ & $33.33(2 / 6)$ & $33.33(1 / 3)$ & $28.57(2 / 7)$ & $40.00(2 / 5)$ & $33.33(3 / 9)$ \\
$X^{2}$ & 12.012 & 12.012 & 20.091 & 9.842 & 12.012 \\
$\mathrm{P}$ & $<0.001$ & $<0.001$ & $<0.001$ & $<0.001$ & $<0.001$ \\
\hline
\end{tabular}

Table 6. Comparison of the diagnostic results of chronic obstructive pulmonary disease based on the two diagnostic methods.

\begin{tabular}{cccccc}
\hline Group & Sensitivity & Specificity & Positive predictive value & Negative predictive value & Diagnostic accuracy \\
\hline Observation group $(\mathrm{n}=8)$ & $70.00(7 / 10)$ & $50.00(2 / 4)$ & $88.89(8 / 9)$ & $66.67(4 / 6)$ & $64.29(9 / 14)$ \\
Control group $(\mathrm{n}=8)$ & $40.00(4 / 10)$ & $25.00(1 / 4)$ & $55.56(5 / 9)$ & $33.33(2 / 6)$ & $35.71(5 / 14)$ \\
$\chi^{2}$ & 14.871 & 12.092 & 15.872 & 12.012 & 14.831 \\
$\mathrm{P}$ & $<0.001$ & $<0.001$ & $<0.001$ & $<0.001$ & $<0.001$ \\
\hline
\end{tabular}

Table 7. Comparison of the diagnostic results of pneumothorax based on the two diagnostic methods.

\begin{tabular}{cccccc}
\hline Group & Sensitivity & Specificity & Positive predictive value & Negative predictive value & Diagnostic accuracy \\
\hline Observation group $(\mathrm{n}=6)$ & $71.43(5 / 7)$ & $66.67(2 / 3)$ & $77.78(7 / 9)$ & $60.00(3 / 5)$ & $60.00(6 / 10)$ \\
Control group $(\mathrm{n}=5)$ & $42.86(3 / 7)$ & $33.33(1 / 3)$ & $44.44(4 / 9)$ & $40.00(2 / 5)$ & $30.00(3 / 10)$ \\
$\chi^{2}$ & 14.761 & 12.012 & 16.982 & 9.631 & 14.779 \\
$\mathrm{P}$ & $<0.001$ & $<0.001$ & $<0.001$ & $<0.001$ & $<0.001$ \\
\hline
\end{tabular}

in China, real-time bedside ultrasonography has been extensively used in the diagnosis of patients with acute dyspnea, especially in the etiologic analysis and diagnosis, with good results [9]. Real-time bedside ultrasonography is not only rapid in terms of diagnosis, but also simple in operation. Moreover, real-time 
bedside ultrasonography can be used to acquire a high accuracy rate on imaging data, providing important guidance for the clinical diagnosis and treatment of acute dyspnea [10]. Real-time bedside ultrasonography also reveals the conditions of the respiratory tract in real-time, facilitating the rapid identification of the cause of dyspnea and the ability to formulate accurate treatment regimens, thereby improving the survival of the patients [11]. In this study, the examination duration of patients receiving real-time bedside ultrasonography was 19.21 $\pm 2.65 \mathrm{~min}$, which was significantly shorter than that of routine examinations $(46.89 \pm 5.82 \mathrm{~min}, \mathrm{P}<0.05)$. Although the cost of medical examination via real-time bedside ultrasonography (CN¥785.05 \pm 8.05$)$ was higher than that of routine examinations (CN¥781.00 \pm 7.03 ), no significant difference was found between the two diagnostic methods. A study of Gu et al. [12] also showed that the examination duration of patients who received real-time bedside ultrasonography $(19.31 \pm 3.98 \mathrm{~min})$ was significantly shorter than that of patients who received routine examinations $(55.89 \pm 4.96 \mathrm{~min})$. Similarly, in the same study, the cost of medical examination of the real-time bedside ultrasonography (CN¥786.75 \pm 8.36) was higher than that of the routine examinations (CN¥783.51 \pm 9.88$)$, while no significant difference in cost was found between the two diagnostic methods, which is consistent with our findings. These results suggest that realtime bedside ultrasonography for the diagnosis of acute dyspnea provides accurate guidance for the analysis of etiologies, accelerated the etiological diagnosis, and facilitated early treatment of the patients.

BNP is a type of neurohormone in the heart, which is mainly secreted by atrial and ventricular cardiomyocytes. Under normal circumstances, when the heart pressure is overloaded, and the blood volume of the heart continues to increase, the ventricular cardiomyocytes will secrete a high level of BNP, which not only has a diuretic effect, but also promotes the dilation of blood vessels, inhibits the activity of the sympathetic nerve system and the renin-angiotensin-aldosterone system, and effectively promotes the proliferation of endothelial cells. Changes in BNP levels accurately reflect changes in ventricular function, as do levels of LVEF and LVED. Some researchers have shown that the higher the BNP level and the lower the LVEF, the more severe the dyspnea. Moreover, BNP is an important hemodynamic index that accurately reflects the left ventricular end-diastolic pressure and provides a basis for relatively accurate clinical diagnosis of acute dyspnea [13]. The results of the current study showed no significant differences in LVEF, LVED, or BNP levels between the two diagnostic methods, which may be due to the relatively small number of patients included in this study.

Additionally, in terms of diagnostic results, real-time bedside ultrasonography had significantly higher diagnostic sensitivity, specificity, positive predictive value, negative predictive value, and diagnostic accuracy for various causes of acute dyspnea compared to routine examinations $(\mathrm{P}<0.05)$. The study of Sun et al. [14] also showed that real-time bedside ultrasonography had significantly higher diagnostic sensitivity, specificity, positive predictive value, negative predictive value, and diagnostic accuracy for various causes of acute dyspnea compared to 
routine examinations $(\mathrm{P}<0.05)$. These findings are consistent with our findings and further confirm that real-time bedside ultrasonography has both high sensitivity and specificity for the etiologic diagnosis of acute dyspnea. We also noted that pneumonia caused significant changes in the subpleural nodules and pleura, resulting in some signs of consolidation in the lungs, such as anechoic areas in the chest, hepatization, and shred sign. These tiny lesions are difficult to capture by conventional X-ray examination [15]; however, real-time bedside ultrasonography accurately captured the information on these tiny lesions and provided accurate guidance for disease diagnosis [16]. After the onset of pulmonary embolism, pulmonary artery become widened, pulmonary artery pressure is increased, and the volume of the right ventricle is increased. The information obtained by conventional X-ray examination is limited, but real-time bedside ultrasonography reveals the indicators of pulmonary function in real time. The information obtained from real-time bedside ultrasonography is more comprehensive, assisting the accurate diagnosis of pulmonary embolism [17]. After the onset of acute cardiogenic pulmonary edema, the systolic function of the left ventricle is weakened, increasing the left ventricular end-diastolic pressure, which frequently leads to complications, including pulmonary congestion and pulmonary edema. The adoption of convention examination requires a relatively long waiting time, and the accuracy of the diagnosis is not guaranteed. The implementation of real-time bedside ultrasonography can continuously scan and diagnose the lateral and anterior chest walls of patients with cardiogenic dyspnea. Moreover, images obtained using real-time bedside ultrasonography show a gravity-dependent bilateral and uniform distribution of B-lines. Under normal circumstances, the conditions of patients who fail to receive timely and effective treatment after the disease onset gradually worsen with time, and the density of B-lines distributed in the images increases [18]. Clinical research has shown that real-time bedside ultrasonography can also be used to analyze the influencing factors of the hemodynamics of some patients with acute dyspnea to provide supportive information for clinical diagnosis [19]. For example, information on ventricular wall thickening, slender inferior vena cava, systolic and diastolic function, and heart chamber size may assist a more accurate determination of the etiology and avoid the challenge of follow-up treatment or other adverse events caused by inaccurate diagnosis of the etiology [20].

\section{Conclusion}

In conclusion, the application of real-time bedside ultrasonography for the etiologic diagnosis of acute dyspnea required a shorter examination duration and achieved a higher diagnostic accuracy, providing accurate guidance for the clinical treatment of acute dyspnea. Therefore, we suggest a more frequent application of this technique in the clinic.

\section{Conflicts of Interest}

The authors declare no conflicts of interest regarding the publication of this paper. 


\section{References}

[1] Zhang, S.G., Wang, Q., Hou, J., et al. (2011) Value of Plasma B-Type Natriuretic Peptide Precursor in Differential Diagnosis of Dyspnea in Elderly Patients. Journal of Taishan Medical College, 32, 257-259.

[2] Yan, C.Y., Jiang, L.Y., Ye, X.D., et al. (2017) Value of NT-proBNP Level in Differential Diagnosis of Acute Dyspnea. Lingnan Journal of Emergency Medicine, 22, 125 126.

[3] Shao, L.W., Han, Y., Wang, Z.K., et al. (2004) Diagnostic Value of Bedside Immediate Brain Natriuretic Peptide Detection in Dyspnea. Chinese Journal of Cardiovascular Diseases, 32, 1123-1125.

[4] Hu, X.L. and Liu, C.X. (2016) Study on the Application Value of Bedside Immediate Detection of Brain Natriuretic Peptide in Patients with Acute Dyspnea. China Medical Guide, 18, 871-872.

[5] Qiu, R.X. and Liu, J. (2019) Diagnosis and Differential Diagnosis of Respiratory Distress Syndrome of Newborn and Wet Lung Using Lung Ultrasonography. Chinese Pediatric Emergency Medicine, 26, 579-582.

[6] Wei, K.Q., Zhang, N. and Liang, X.X. (2020) Clinical Value of Brain Natriuretic Peptide in the Diagnosis of Emergency Dyspnea. Health Vision, 28, 34.

[7] Huang, Q.Y. (2011) Diagnostic Value of Plasma Brain Natriuretic Peptide in Acute Dyspnea. Modern Medicine and Health, 27, 1845-1846.

[8] Qiang, J.T. and He, J.W. (2020) Effect of Severe Ultrasound Rapid Management Program on Etiological Diagnosis of Acute Dyspnea or Hemodynamic Instability in RICU Patients. International Journal of Respiration, 40, 1154-1159.

[9] Liu, X., Hang, G., Hu, J.H., et al. (2019) Analysis of Etiology, Characteristics and Risk Factors of 1135 Cases of Neonatal Dyspnea. China Medical Herald, 16, 100-103.

[10] Zhang, J.L., Feng, N.F. and Zhang, J. (2016) Preliminary Analysis of the Value of N-Terminal B-Type Brain Natriuretic Peptide Precursor in Judging the Etiology of Acute Dyspnea. Application of Modern Medicine in China, 10, 20-22.

[11] Ma, H.J. (2017) Etiological Analysis of 166 Cases of Non Respiratory Diseases Due to Dyspnea in Pediatric Emergency Department. Chinese Community Physician, 33, 37-39.

[12] Gu, H.P. (2019) Diagnostic Value of Bedside Immediate Detection of Brain Natriuretic Peptide in Patients with Heart Failure. Electronic Journal of Integrated Traditional Chinese and Western Medicine on Cardiovascular Disease, 7, 103.

[13] Chen, Y.E and Wu, J.W. (2019) Evaluation of Clinical Value of Brain Natriuretic Peptide in the Diagnosis of Emergency Dyspnea. Medical Diet and Health, 3, 35.

[14] Sun, T., Bao, W.H., Zhang, Y., et al. (2020) Value of Bedside Real-Time Ultrasound in Etiological Diagnosis of Acute Dyspnea. Medical Information, 33, 174-176.

[15] Ke, B.S. (2017) Analysis of Brain Natriuretic Peptide Levels in Patients with Pulmonary Dyspnea and Cardiogenic Dyspnea. Grassroots Medical Forum, 21, 3015-3016.

[16] Zhang, H.W., Zou, Y.S. and Guo, Y.C. (2017) Application Value of Brain Natriuretic Peptide in Differential Diagnosis of Patients with Acute Dyspnea. Mother Baby World, 17, 19-20.

[17] Man, N. (2017) Diagnostic Value of Bedside Rapid Detection of N-Terminal pro Brain Natriuretic Peptide in Elderly Patients with Acute Dyspnea. World Clinical Medicine, 11, 39.

[18] Xiong, L. (2016) Clinical Application of N-Terminal-B-Type pro Brain Natriuretic 
Peptide (NT proBNP) in the Differential Diagnosis of Emergency Senile Dyspnea. China Health Nutrition, 26, 68-69.

[19] Wang, Z., Li, H.Y. and Cai, L.X. (2016) Clinical Study of Brain Natriuretic Peptide in Differential Diagnosis of Acute Dyspnea in Children. Jiangxi Medicine, 51, 694695.

[20] Ding, C. (2019) Evaluation of Brain Natriuretic Peptide in Middle-Aged Patients with Dyspnea and Heart Failure. Medical Frontier, 9, 72-73. 